\title{
Signal Processing Techniques for Transmission Impairments Compensation in Optical Systems
}

\author{
H.M. Salgado ${ }^{1}$, J.M.B. Oliveira ${ }^{1}$, L.M. Pessoa ${ }^{1}$ and I. Darwazeh ${ }^{2}$ \\ ${ }^{1}$ INESC Porto, Faculdade de Engenharia, Universidade do Porto, Portugal \\ ${ }^{2}$ University College London, $U K$ \\ \{hsalgado;jmbo;lpessoa\}@inescporto.pt,i.darwazeh@ee.ucl.ac.uk
}

\begin{abstract}
We discuss recent developments in signal processing techniques for post-compensation of fiber dispersion and nonlinear distortion in both coherent and radio-over-fiber optical systems.
\end{abstract}

Keywords: OFDM, channel estimation, nonlinear analysis, radio-over-fiber, coherent systems, backpropagation.

\section{INTRODUCTION}

Signal processing in optical systems is playing an important role in mitigating fiber transmission impairments due to the increasing transmission rates and bandwidth demand. The mitigation of fibre nonlinearity using digital back-propagation (BP) is currently a highly debated topic [1][2]. In this context it is of interest to evaluate the performance and complexity of different modulation formats. On the other hand, Radio-over-Fiber (RoF) is a technology that is being increasingly used to transport and deliver radio signals from remote antenna units (RAU) to a central station (CS) [3]. Yet, these systems also suffer from specific impairments which include the nonlinearities associated with various optical components such as electro-optic converters.

Here we discuss recent results on the BP algorithm to evaluate the performance of both single-carrier (SC) and orthogonal frequency division multiplexing (OFDM) modulations in coherent optical systems as well as new channel estimation techniques, especially for multicarrier modulation schemes, suitable for RoF systems.

\section{COMPENSATION OF NONLINEAR EFFECTS THROUGH DIGITAL BACKPROPAGATION}

The signal that propagates through a standard single mode fibre (SSMF) is affected by both dispersion and nonlinearity, according to the Non Linear Schrodinger Equation (NLSE) [4]. In the linear transmission regime, the capacity increases with the launch power, whereas in the nonlinear regime, the capacity is reduced, because fibre nonlinearity scales with the square of the power while signal-to-noise ratio (SNR) grows linearly with the power. The best method known for fibre nonlinearity mitigation is BP, which consists in passing the received signal through a virtual fibre with opposite signs of dispersion and nonlinearity, yielding an estimate of the transmitted signal. In the absence of noise and limited computation power, and if the characteristics of the transmission channel are known, this technique can obtain exactly the transmitted signal. While BP was first used in pre-compensation schemes [2], the availability of the full electric field in a coherent optical receiver allows for BP to be used as a post-compensation technique. Since the BP algorithm works with the electric field of the signal, it can be applied to single and multicarrier signals. BP calculates a solution for the NLSE, which is generally numerically solved using the Split Step Fourier Method (SSFM).

We study the nonlinear performance of two main candidates for long haul optical transmission, Polarization Division Multiplexed Quadrature Phase Shift Keying (PDM-QPSK) and Coherent Optical OFDM (CO-OFDM), focusing on both dispersion management and computational requirements analysis. However, in our analysis we neglect the effect of polarization for simplicity.

\subsection{Backward Propagation Algorithm}

There are two commonly used ways of applying the SSFM, the non-iterative asymmetric approach and the iterative, more accurate and computationally intensive symmetric approach [2]. While the symmetric approach consists in evaluating the nonlinear operator in the middle of the step, by splitting the dispersion operator in two parts requiring an iterative approach, in the asymmetric approach, the need for iteration is removed by approximating the integral of the nonlinear operator with a one-sided rectangular rule [5]. The linear operator (mitigation of dispersion) is evaluated in the frequency domain (1), while the nonlinear operator is more effectively applied as a phase rotation in the time domain (2).

$$
F F T\{\exp (h \hat{D}) E(z, t)\}=\exp \left(\left(-\frac{\alpha}{2}+j \frac{\beta_{2}}{2} \omega^{2}\right) h\right) E(z, \omega)
$$

The Fast Fourier Transform (FFT) and respective IFFT are used to switch between both domains, which becomes the most computationally expensive operation in the whole algorithm.

$$
\exp (h \hat{N}) E(z, t)=\exp \left(j \xi \gamma|E(z, t)|^{2} h\right) E(z, t)
$$


The parameter $0 \leq \xi \leq 1$, is the amount of nonlinearity compensated, reflecting the uncertainty of the signal amplitude used to undo the nonlinear phase rotations. It is a system parameter that depends on the launched power, dispersion map, oversampling rate and number of steps used for BP. This parameter is found by numerical simulation, and generally is small for low residual dispersion per span (RDPS), and approaches 1 for dispersion unmanaged transmission. Additionally, the parameter tends to 1 for increasing number of steps of BP.

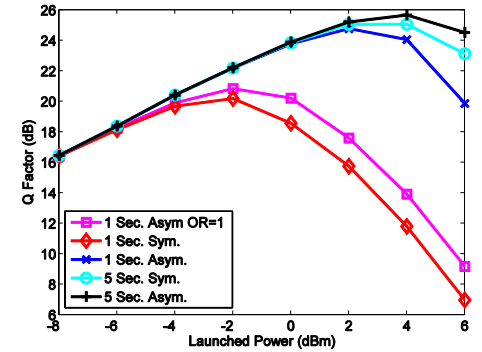

(a) OFDM $100 \%$ RDPS

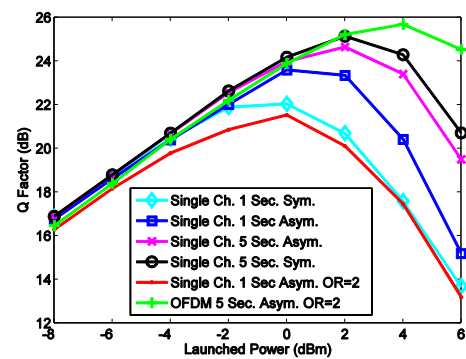

(b) SC vs OFDM $100 \%$ RDPS

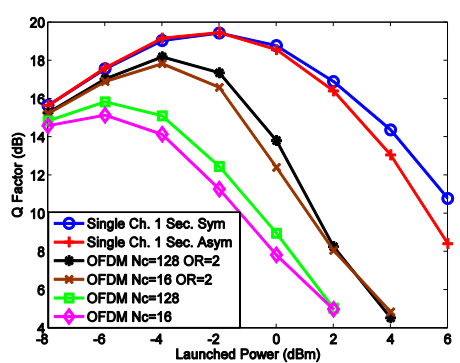

(c) SC vs OFDM $5 \%$ RDPS

\subsection{Simulations Results}

We consider a $10 \mathrm{GS} / \mathrm{s}$ long haul transmission link comprised by 25 spans of $80 \mathrm{~km} \mathrm{SMF}$ and variable length of $\mathrm{DCF}$, having dispersion coefficient of 17 and $-80 \mathrm{ps} / \mathrm{nm} / \mathrm{km}$, nonlinear coefficient of 1.3 and $5.3 \mathrm{~km}^{-1} \mathrm{~W}^{-1}$, and loss of 0.2 and $0.6 \mathrm{~dB} / \mathrm{km}$, respectively. We analyse two dispersion management configurations: $100 \%$ RDPS corresponding to dispersion unmanaged transmission, and a dispersion managed link with residual dispersion of $5 \%$ per span. The performance metric used was the $\mathrm{Q}$ factor as detailed in [6]. The oversampling rate (OR) used for single channel transmission was 3 samples per symbol, to avoid aliasing as suggested by the results of [5]. We considered a reference OFDM signal, having $N_{c}=128$ carriers, with $N_{u}=88$ of them being used for transmission of QPSK symbols, and a guard interval of $1 / 8$ of the observation period.

In Figure 1(a), we can observe the performance of OFDM against a sweep in launched power (LP) from $8 \mathrm{dBm}$ to $+6 \mathrm{dBm}$. We can see that for an OR of 1 , the performance is very poor. However, if the OR is increased to 2 , much improvement is obtained. We also compare the results in terms of number of sections (or steps) used in the BP algorithm, and whether symmetric or asymmetric SSFM is used. We verified that after 5 sections for both symmetric and asymmetric approaches, the performance stabilizes. Increasing after that point is not worth in terms of performance gain/computational effort. We may observe that for a single section, the asymmetric algorithm provides much better performance. For 5 sections both approaches provide good performance, but the difference for the asymmetric single section case is less than $2 \mathrm{~dB}$ at $+4 \mathrm{dBm}$.

In Figure 1(b) we can observe that the performance of OFDM is better than SC, for a similar number of sections and is nearly the same when 1 section is used for OFDM and 5 sections for SC. We have additionally plotted the result of SC with an OR of 2 and verified that it is indeed very poor compared to $O R=3$ with more than $2 \mathrm{~dB}$ of penalty at the optimum launched power of $0 \mathrm{dBm}$. Regarding the scenario of 5\% RDPS in Figure 1(c), OFDM has much poorer behaviour than SC, which can be attributed to the high peak to average power ratio (PAPR) when compared to SC, which is problematic when phase adds coherently from span to span, a phenomenon called phase matching, arising from dispersion management. Additionally, the case of OFDM with $N_{c}=16$ is also shown, to validate the fact that the reduced PAPR of this modulation allows for the performance to approximate that of OFDM $N_{c}=128$ for increasing LP.

\section{CHANNEL ESTIMATION WITH OFDM SIGNALLING IN RADIO-OVER-FIBER SYSTEMS}

In multicarrier modulation schemes, like OFDM, the signal is composed by a set of narrowband subcarriers, each usually having a frequency bandwidth narrower than the coherence of the radio channel. As a consequence, the frequency response of the channel is considered to be flat over each subcarrier. Therefore, channel estimation and equalization can be performed by simple zero-forcing (ZF) techniques [7][8]. However, ZF estimators do not take into account the correlation between subcarriers from multipath and nonlinear distortion.

Here, we derive the structure and study the performance of the linear minimum mean-squared error (MMSE) channel estimator when considering nonlinearly distorted OFDM signalling.

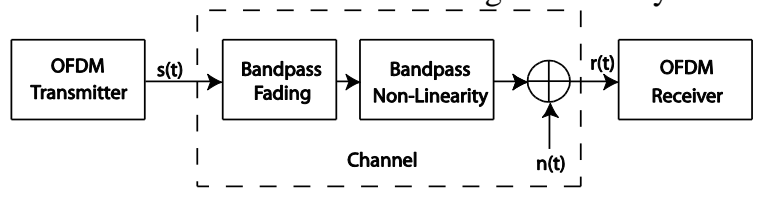

Figure 2: Equivalent baseband OFDM wireless communications model.

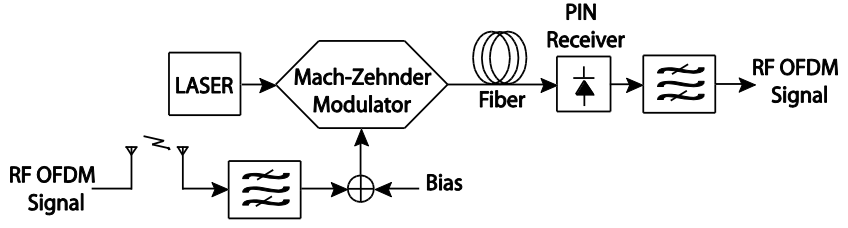

Figure 3: Radio-over-Fiber uplink system. 


\subsection{System Model}

We consider the OFDM communications system model shown in Figure 2, where $s(t)$ is the complex envelope of the transmitted OFDM signal, $r(t)$ is the complex envelope of the received OFDM signal, $n(t)$ is the complex additive white Gaussian noise (AWGN) with power spectral density $N_{0}$. Note that this system can represent a typical RoF uplink system as it is shown in Figure 3. Here the bandpass multipath fading, that represents the radio channel between the wireless device and the RAU, is followed by the bandpass nonlinearity which represents the electro-optic modulator and the additive white Gaussian noise (AWGN) source from the PIN receiver. The transmit signal is distorted both by a time-dispersive (multipath) and nonlinear channels. The impulse response of the time-dispersive channel is given by [8]

$$
h(t)=\sum_{i=0}^{M-1} h_{i} \delta\left(t-\tau_{i}\right)
$$

where $h_{i}$ and $\tau_{i}$ are the different complex gains and path time delays, respectively, $M$ is the total number of paths and $\delta($.$) is the Kronecker delta. The complex gains, h_{i}$, are wide-sense stationary narrow-band complex Gaussian processes uncorrelated with respect to each other.

At the receiver side, the received OFDM symbols can be obtained by applying a set of filters matched to every frequency components [9]. Finally, an estimate of the transmitted OFDM symbols can be obtained by applying a ZF equalization to the received OFDM symbol, i.e., $\hat{S}_{k, n}=R_{k, n} / \hat{H}_{k, n}$, where $\hat{H}_{k, n}$ is the estimate of the frequency response of the multipath channel estimate in time slot $k$ and subcarrier $n$.

\subsection{LMMSE Channel Estimator}

We now consider the structure of the LMMSE channel estimator for nonlinearly distorted OFDM signals in AWGN. The channel estimator will process the distorted OFDM signal in a linear fashion in order to optimize a performance metric, namely, the mean-squared error (MSE), making full use of subcarrier correlation and of redundant information from intermodulation products (IMP).

We take the estimator to consist of a bank of $N$ linear filters with impulse responses $u_{n}(t), n=0, \ldots, N-1$, that produce estimates of the channel frequency response. The objective is to determine the set of linear filters impulse response that minimize the MSE given by [10]

$$
\xi=E\left\{\left|\mathbf{H}_{k}-\hat{\mathbf{H}}_{k}\right|^{2}\right\}=\sum_{n=0}^{N-1} E\left\{H_{k, n}-\left[u_{n}\left(t_{k}\right) * r\left(t_{k}\right)\right]^{2}\right\}
$$

where $\mathbf{H}_{\mathrm{k}}$ and $\hat{\mathbf{H}}_{\mathrm{k}}$ are the vectors of the channel frequency response and of estimates of channel frequency response, respectively, in time slot $k$.

Assuming now that the nonlinearity exhibits nonlinear behaviour of maximum order $L$ ( $L$ is odd), using calculus of variations [11] we find that the set of complex functions $u_{n}(t)$ that minimize the MSE is given by

$$
u_{n}(t)=\sum_{i=i_{\min }}^{i_{\max }} \beta_{i, n} g_{i}^{*}\left(t_{0}-t\right), n=0, \cdots, N-1,
$$

where $i_{\min }=-(L-1)(N-1) / 2, i_{\max }=(L+1)(N-1) / 2$, and the coefficients $\beta_{i, n}$ correspond to the solution of the following set of linear equations

$$
\sum_{i=i_{\min }}^{i_{\max }} \beta_{i, n} E\left\{Z_{i} Z_{m}^{*}\right\}+2 N_{0} \beta_{m, n}=E\left\{H_{k, n} Z_{m}^{*}\right\}, \text { where } Z_{m}=\int_{k T+T_{C P}}^{(k+1) T} y(t) g_{m}^{*}(t-k T) d t
$$

and $m=-(L-1)(N-1) / 2, \ldots,(L+1)(N-1) / 2$.

In order to derive the impulse responses $u_{n}(t)$ that minimize the MSE given by (4) and the expectations in (6), a mathematical approach analogous to the one performed by Oliveira et al in [9] can be performed.

Interestingly, the LMMSE channel estimator structure for nonlinearly distorted OFDM signals in the presence of AWGN can be seen as a set of linear filters with impulse responses $u_{n}(t)$, as well as a structure consisting of a set of correlators or a set of filters matched to every possible frequency components in the nonlinearly distorted OFDM signal followed by a simple linear transformation solely defined by the coefficients $\beta_{i, n}$.

\subsection{Case study: Radio-over-Fiber Wireless Networks}

We consider the RoF intensity modulation/direct detection (IM/DD) uplink system shown in Figure 3. For simulation results the optical fiber is assumed to be ideal, the laser to have an optical power of $0 \mathrm{dBmW}$, the photodiode to have a responsivity $R=1 \mathrm{~A} / \mathrm{W}$ and the power spectral density of the PIN receiver's thermal noise, which is assumed to be white and Gaussian, $N_{0}$.

In this partial application we examine the performance of the LMMSE channel estimator for nonlinearly distorted OFDM signals in AWGN. For performance comparison, we have used the Least Squares (LS) channel estimation given by $\hat{H}_{k, n}^{L S}=\left(c_{1} S_{k, n}\right)^{-1} R_{k, n}$ [8]. This LS estimation is a straightforward estimate of the channel 
attenuation of the $n$th subcarrier, obtained by just considering that the channel is linear, and does not exploits or recognizes the correlation between subcarriers.

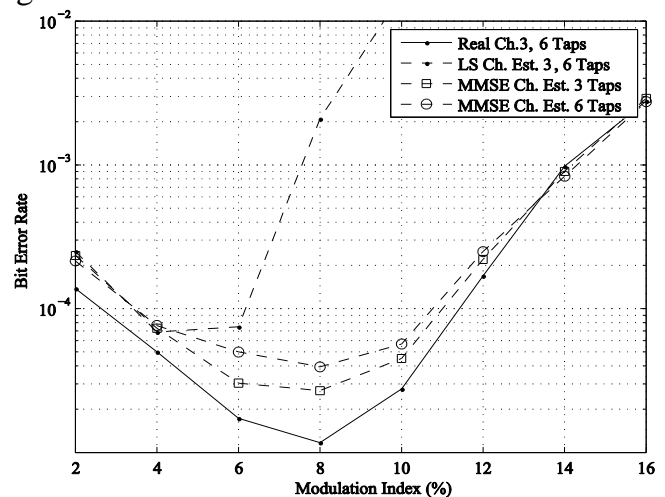

Figure 4: BER of a 16 subcarrier OFDM signal vs modulation index for $N_{0}=2.45 \times 10^{-15} \mathrm{~W} / \mathrm{Hz}$.

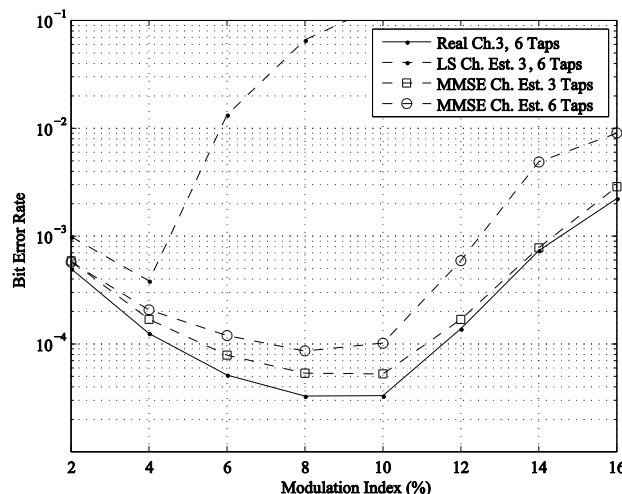

Figure 5: BER of a 48 subcarrier OFDM signal vs modulation index for $N_{0}=2.45 \times 10^{-15} \mathrm{~W} / \mathrm{Hz}$.

Figure 4 and Figure 5 show the performance of the LS and LMMSE channel estimators with ZF equalization for channels consisting of three and six paths for 16 and 48 subcarriers, respectively. It can be seen that the LMMSE estimative outperforms the LS estimative. In fact, channel estimation that takes advantage of the correlation between subcarriers due to multipath and intermodulation products, presents performances that tend to the hypothetical and ideal case when the multipath channel is known and no estimation is needed. Another interesting result is that the difference between estimatives increases with the number of subcarriers, which comes from the increase of IMP and, consequently, the lower performance of the LS channel estimation.

\section{CONCLUSIONS}

In this paper, we used BP to evaluate the performance of both SC and OFDM modulations in the nonlinear regime of coherently detected signals. OFDM has shown higher tolerance for dispersion unmanaged transmission, losing this advantage for dispersion managed systems. We also derived the structure of the linear MMSE channel estimator for nonlinearly distorted OFDM signals which is shown to be suitable for the uplink of radio-over-fiber systems. Simulation results have shown that the LMMSE channel estimator outperforms the LS channel estimator and that its difference increases with the number of OFDM subcarriers.

\section{ACKNOWLEDGMENT}

This work was supported by FCT under the project "Design and Optimisation of WDM Millimetre-Wave FibreRadio Systems" (PTDC/EEA-TEL/68974/2006) and in the framework of programme POCTI/FEDER with grant REEQ/1272/EEI/2005. João Oliveira and Luís Pessoa also acknowledge support from FCT through a PhD grant.

\section{REFERENCES}

[1] L.M. Pessoa, H.M. Salgado, I. Darwazeh, "Simplified backpropagation equalization in WDM coherent polarization multiplexed systems", Int. Conference on Transparent Optical Networks, 2009.

[2] E.M. Ip and J.M. Kahn, "Fiber Impairment Compensation Using Coherent Detection and Digital Signal Processing," Journal of Lightwave Technology, vol. 28, pp. 502-519, 2010.

[3] H. Ogawa, D. Polifko, and S. Banba, "Millimeter-wave fiber optics systems for personal radio communication," IEEE Trans. Microwave Theory Tech., vol. 40, no. 12, pp. 2285-2293, 1992.

[4] G. P. Agrawal, Nonlinear Fiber Optics, 2nd ed. London, U.K.: Academic, 1995.

[5] E.M. Ip and J.M. Kahn, "Compensation of Dispersion and Nonlinear Impairments Using Digital Backpropagation” J. of Lightwave Technol., vol. 26, pp. 3416-3425, 2008.

[6] A.J. Lowery, L.B. Du, and J. Armstrong, "Performance of Optical OFDM in Ultralong-Haul WDM Lightwave Systems" J. Lightwave Technol., vol. 25, pp. 131-138 , 2007.

[7] D. Lowe, and X. Huang, "Adaptive low-complexity MMSE channel estimation for OFDM," Int. Symposium on Communications and Information Technologies, Thailand, 2006.

[8] J. Beek, O. Edfors, M. Sandell, P. Borjesson, and S. Wilson, "On channel estimation in OFDM systems," Vehicular Technologies Conference, Chicago, 1995.

[9] J. Oliveira, M. Rodrigues, and H. Salgado, "MMSE receivers for nonlinearly distorted OFDM signals," 7th Conference on Telecommunications, Portugal, 2009.

[10] S. Benedetto and E. Biglieri, Principles of Digital Transmission: With Wireless Applications, Kluwer Academic Publishers, 1999.

[11] R. Weinstock, Calculus of Variations. With Applications to Physics and Engineering. New York, 1974. 